\title{
Revegetation in Dead Dicranopteris (Gleicheniaceae) Fern Patches Associated with Hawaiian Rain Forests ${ }^{1}$
}

\author{
Peter A. Follett, ${ }^{1,2,3,4}$ Puanani Anderson-Wong, ${ }^{3,5}$ M. Tracy Fobnson, ${ }^{3,6}$ and Vincent P. Fones ${ }^{3,7}$
}

\begin{abstract}
Dieback of Dicranopteris linearis (Burm. f.) Underwood on wet, open valley slopes and ridgelines of Maui, O'ahu, and Kaua'i has been attributed to feeding by the introduced leafhopper Sophonia mufofascia Kuoh \& Kuoh. We studied early plant succession at a variety of low-elevation $D$. linearis dieback sites to assess the vulnerability of these disturbances to invasion by nonnative weeds. Dead patches of $D$. linearis were colonized by both native and alien plant species; the number and assemblage of colonizing plant species was site specific. Clidemia birta (L.) D. Don and Nepbrolepis multiflora (Roxb.) Jarrett ex C. Morton were the most common invasive species colonizing and spreading in dieback patches. Recolonization of dead patches by live $D$. linearis spreading from the margins was also common. In a simulated fern decomposition study, seedling germination increased as the depth of the thicket decreased. Fern dieback may enhance regeneration of the native tree Acacia koa A. Gray.
\end{abstract}

The uluhe or false staghorn fern, Dicranopteris linearis (Burm. f.) Underwood, is an important gap species in the wetter Hawaiian forests and watersheds (Merlin 1995). Mueller-Dombois and Fosberg (1998) recognized $D$. linearis as forming a distinct indigenous vegetation type, designated as Dicranopteris fernlands, in the Hawaiian rain forest. As such, it plays an important role in structuring the plant community in many habitats. In the Tropics, ferns such as D. lin-

${ }^{1}$ Manuscript accepted 9 March 2003.

2 Corresponding author.

${ }^{3}$ Department of Entomology, University of Hawai'i at Mānoa, Honolulu, Hawai'i 96822.

${ }^{4}$ Current address: USDA-ARS, U.S. Pacific Basin Agricultural Research Center, P.O. Box 4459, Hilo, Hawai'i 96720 (E-mail: pfollett@pbarc.ars.usda.gov).

${ }^{5}$ Current address: Department of Botany, University of Hawai'i at Mānoa, Honolulu, Hawaíi 96822.

${ }^{6}$ Current address: Hawai 1 i Volcanoes National Park Quarantine Facility, USDA Forest Service, P.O. Box 236, Volcano, Hawai'i 96785.

${ }^{7}$ Current address: Department of Entomology, Tree Fruit Research and Extension Center, Washington State University, 1100 North Western Avenue, Wenatchee, Washington 98801.

Pacific Science (2003), vol. 57, no. 4:347-357

(C) 2003 by University of Hawai'i Press

All rights reserved earis are often the early colonists of severely disturbed sites, such as landslides (Wardlaw 1931, Scott 1969, Guariguata 1990), lava flows (Atkinson 1970), and abandoned farmland (Kochummen and Ng 1976). Thicketforming, vinelike ferns such as $D$. linearis play an important role in stabilizing soils, particularly in areas of steep slope and high rainfall (Scott 1969). They also have a dynamic function in early and late successional development in the Hawaiian rain forest before Metrosideros ('ohi'a lehua) canopies close and when canopies open up due to tree dieback (Mueller-Dombois 2000). These ferns spread laterally by extensive rhizomes that grow at or just beneath the soil surface and periodically send up fronds; a single rachis grows one stage at a time, climbing on the dead and decaying fern stems in monotypic stands, or using the support of other plants in mixed stands (Holttum 1957).

In the late 1980 s, many monotypic stands of $D$. linearis in open areas along valley slopes and ridgelines on the islands of Maui, $\mathrm{O}^{6} \mathrm{ahu}$, and Kaua' $i$ began to die, leaving behind thickets of dead stems (Palmer 1993). Dieback of $D$. linearis coincided with the accidental introduction and proliferation of the two-spotted leafhopper, Sopbonia rufofascia Kuoh \& Kuoh. Sopbonia rufofascia was first 
detected in 1988 on $\mathrm{O}^{\prime}$ ahu on fiddleleaf fig, Ficus lyrata Warb., and quickly spread to all the major Hawaiian islands (Heu and Kumashiro 1989). It now has a host list of over 300 plant species in 80 families (Fukada 1996), including ferns, grasses, shrubs, and trees. Laboratory and field studies implicate $S$. rufofascia as the cause of dieback of $D$. linearis (Jones et al. 2000). However, on the island of Hawai'i, where S. rufofascia also occurs and is trapped in high numbers in D. linearis (Jones et al. 2000, Yang et al. 2000) no dieback has been observed, suggesting that other factors must have contributed to the process.

Stands of $D$. linearis in Hawai'i can range in height from a few centimeters to a few meters (Kepler 1984). Dense thickets can prevent the growth of other plants (Walker 1994, Mueller-Dombois 2000). In addition to exposing slopes to soil erosion, the decline and death of stands of $D$. linearis (typically $0.1-0.5 \mathrm{ha}$ ) can radically alter the composition of the plant community through species replacement. In Hawai'i, opportunistic alien weeds, such as Clidemia birta (L.) D. Don (Koster's curse), Citbarexylum caudatum L. (fiddlewood), Nepbrolepis spp. (swordfern), Lantana camera L., Psidium cattleianum Sabine (strawberry guava), Rubus rosifolius Sm. (thimbleberry) and others, can invade disturbed sites and form large monotypic stands, preventing native species replacement (Cuddihy and Stone 1993). We studied early plant succession in a variety of low-elevation $D$. linearis dieback areas to assess the vulnerability of these disturbed sites to invasion by nonnative weeds.

\section{MATERIALS AND METHODS}

\section{Permanent Plots}

The fern dieback sites sampled all occurred within $100 \mathrm{~m}$ of well-maintained hiking trails. Six patches of dead $D$. linearis in the southern Ko'olau Range of $\mathrm{O}^{6}$ ahu were selected to monitor revegetation patterns through time, and plots were established between May and July 1996. Sites were Ironwood and St. Stephen along the Maunawili Trail on the windward side, and MC-steep and MC-flat along the Mānoa Cliff Trail, Pưu Pia in Mānoa Valley, and 'Aihualama along the 'Aihualama Trail on the leeward side (Figure 1). At each site, plots were selected that were primarily dead $D$. linearis stems, with minimal recolonization. Plots were 20 by $10 \mathrm{~m}$, which was approximately one-half the total area of the smallest patch sampled and one-tenth the area of the largest patch. Quadrat and line intercept sampling were done at $0,6,14$, and $27-32$ months to estimate changes in plant species composition and percentage cover (Mueller-Dombois and Ellenberg 1974). Ten $1-\mathrm{m}^{2}$ quadrats were randomly selected and permanently established within each plot at the beginning of the experiment, and on subsequent sampling dates the number of stems and percentage cover of each plant species within each quadrat were determined. In addition, on each sampling date four transects were established across the $10-\mathrm{m}$ width of the plots and plants were scored at $0.5-\mathrm{m}$ intervals along each transect (84 point intercepts per plot) as an alternate estimate of percentage cover for various plant species.

\section{Wayside Vegetation Analysis}

trail transects. Hiking trails can be thought of as transects across the landscape and are useful for vegetation analysis in areas that are otherwise inaccessible due to steep terrain or thick vegetation. The 'Aiea Loop and Maunawili Trails (Ball 2000) were used as transects across two steep valleys on $\mathrm{O}^{6} \mathrm{ahu}$ known to have areas of $D$. linearis dieback to estimate percentage cover of $D$. linearis in those valleys. Sampling was conducted in June 1996 at $100-\mathrm{m}$ intervals along both trails. At each interval, both sides of the trail were sampled using the quadrat-on-a-stick method (a 1- $\mathrm{m}^{2}$ PVC pipe quadrat at the end of a 2-m-long PVC pole). This extendedquadrat device enabled us to sample vegetation beyond the disturbed area bordering the trail. At each sampling interval the quadrat was positioned at points above and below the trail and estimates were made of percentage cover within the quadrat on the ground and in the overstory above. The survey included 

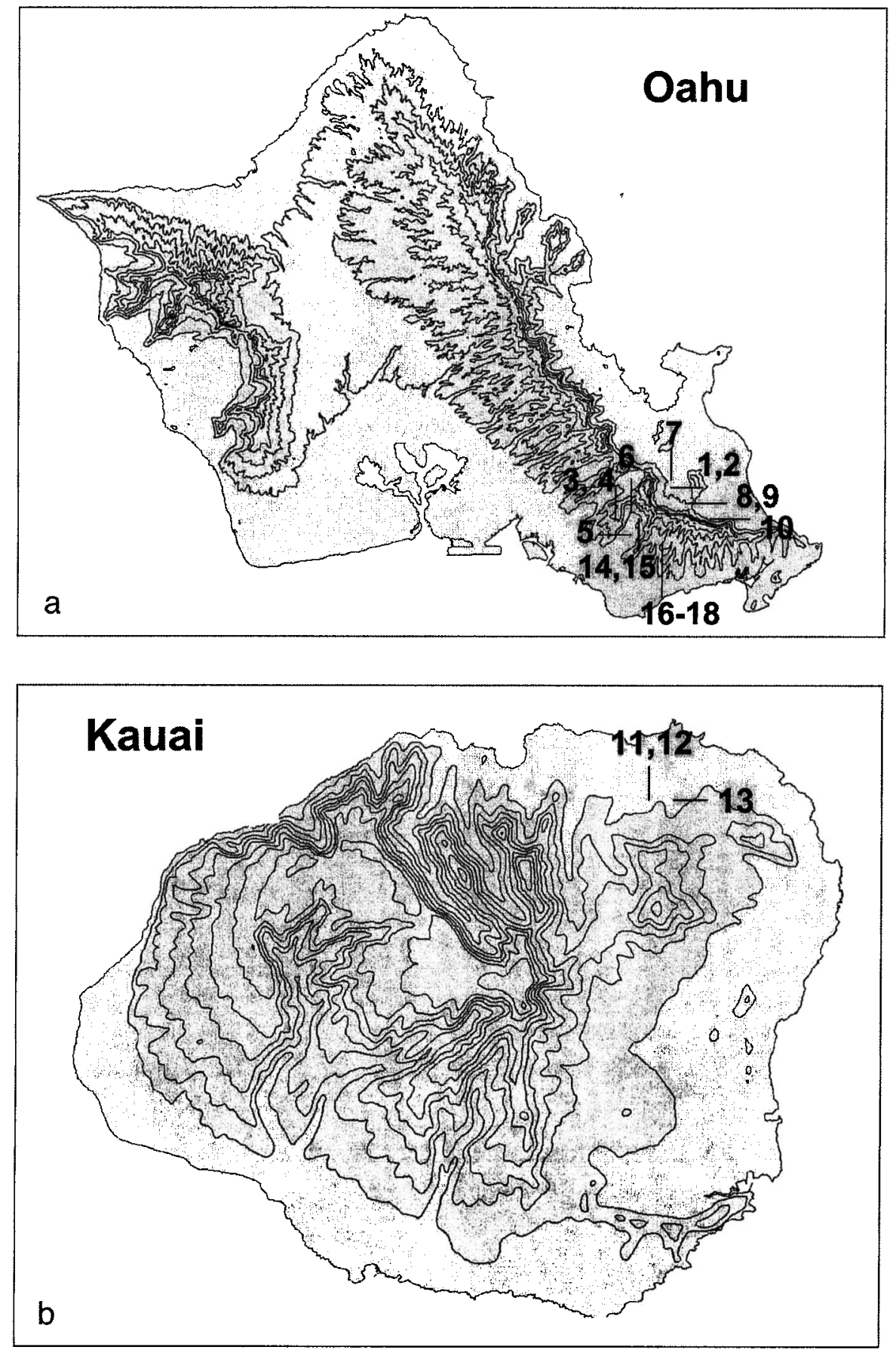

FIGURE 1. Dicranopteris dieback sites sampled on the islands of $\mathrm{O}^{\prime}$ ahu $(a)$ and Kaua $\mathrm{i}(\mathrm{b})$ : 1 , Ironwood; 2, St. Stephen; 3, MC-steep; 4, MC-flat; 5, Pu'u Pia; 6, 'Aihualama; 7, 15 min; 8, MW-site A; 9, MW-site C; 10, 3-mile; 11, Guavakai 1; 12, Guavakai 2; 13, Powerline; 14, Mānoa Falls 1; 15, Mānoa Falls 2; 16, Maúumae 1; 17, Maúumae 2; 18, Mau'umae 3. 
$6.9 \mathrm{~km}$ of the Maunawili Trail and $8 \mathrm{~km}$ of the 'Aiea Loop Trail.

DIEBACK PATCHES. Hiking trails also are the best access to many of the $D$. linearis dieback areas. In addition to the 10 by $20 \mathrm{~m}$ permanent plots, 12 wayside patches of dead $D$. linearis were sampled in June 1997 along four ridges on the islands of $\mathrm{O}^{\prime} \mathrm{ahu}$ and Kaua ${ }^{\circ}$ using point-intercept sampling. Sites were 15 min, $M W$-sites $\mathrm{A}$ and $\mathrm{C}$, and 3 -mile along the Maunawili Trail on the windward side of the southern Ko'olau Range on O'ahu; Guavakai 1 and 2 and Powerline near the Powerline Trail on Kaua'i; Mānoa Falls 1 and 2 along the Mānoa Falls Trail; and Mau'umae 1, 2, and 3 along the Mau'umae Trail on the leeward side of the southern Ko'olau range (Figure 1). Plants were censused every $0.5 \mathrm{~m}$ along a $10-\mathrm{m}$ rope stretched across the patch at three or four (depending on the size of, and access to, the dead patch) randomly selected origins. These patches were typically at a later stage of revegetation compared with the permanent plot sites.

\section{Mulcb Plots}

It was observed in the field that dense thickets of dead $D$. linearis stems completely shaded the soil beneath and suppressed germination of seed bank plants. In 1997-1998, an experiment was conducted to determine the effect of fern decomposition on revegetation. Experimental plots were set up in large areas of dead $D$. linearis that were devoid of live vegetation. Nine $1-\mathrm{m}^{2}$ plots were established at each of three sites in Mānoa Valley on the island of O'ahu. The experimental design involved three treatments that were replicated three times at each site using a randomized block design. Treatments were (1) bare soil (all dead D. linearis plant material removed), (2) mulch (dead D. linearis stems cut into 5 - to 10 -cm pieces), and (3) thicket (dead D. linearis thicket left untouched). Before establishing the treatments, data were taken on thicket depth by systematically measuring thicket depth at six different coordinates within each $1-m^{2}$ subplot and measuring the deepest and shallowest points in the subplot. Seed- ling germination data from the mulch plots were taken at 3 and 9-11 months after the plots were established. Data on plant counts were subjected to analysis of variance after $\log (x+1)$ transformation. Tukey-Kramer's HSD $(\alpha=0.05)$ was used to make paired comparisons between treatment means (SAS Institute 2002).

\section{RESULTS}

The plant species occurring in patches of dead D. linearis sampled from 1995 to 1999 are shown in Table 1 . The most frequently encountered species in addition to $D$. linearis were the exotic species Citbarexylum caudatum, Clidemia birta, Erechtites valerianifolia (Wolf) DC (fireweed), and Nephrolepis multiflora (Roxb.) Jarrett ex C. Morton and the endemic species Acacia koa A. Gray (koa).

\section{Permanent Plots}

Point-intercept and quadrat sampling of the permanent plots showed that all plots were recolonized by both native and invasive species during the study (Table 2). Clidemia birta was the main invasive species in the Ironwood and $\mathrm{Pu}^{6} u \mathrm{Pia}$ plots; in both plots 11-13\% of point-intercept samples were positive for C. birta at the start of the study and this increased to $40 \%$ at $30-31$ months later. Quadrat sampling also showed about a fourfold increase in the percentage cover of $C$. birta in the Ironwood plot after 30 months. Both quadrat and point-intercept sampling showed that $C$. birta was present in all permanent plots at 30-33 months. The next most common invasive species was $C$. caudatum, which was present at low levels in four plots. Dicranopteris linearis was recolonizing five of the six plots, and in the St. Stephen, MC-flat, and 'Aihualama plots it appeared to be increasing toward full recovery. It is interesting that seedlings of the native tree $A$. $k o a$ were germinating in four of the dieback plots at 30-33 months, whereas this native tree typically does not regenerate in undisturbed dense monotypic stands of D. linearis (P.A.F., pers. obs., but see Mueller-Dombois 2000). 
TABLE 1

Plant Species Colonizing Dead Patches of Dicranopteris linearis Sampled on O'ahu and Kaua'i, Hawai $i$, 1995-1999

\begin{tabular}{|c|c|c|c|c|c|c|c|c|c|c|c|c|c|c|c|c|c|c|c|c|}
\hline \multirow[b]{2}{*}{ Species } & \multirow[b]{2}{*}{ Common Name } & \multirow[b]{2}{*}{ Origin $^{a}$} & \multicolumn{18}{|c|}{ Sites $^{b}$} \\
\hline & & & 1 & 2 & 3 & 4 & 5 & 6 & 7 & 8 & 9 & 10 & 11 & 12 & 13 & 14 & 15 & 16 & 17 & 18 \\
\hline \multicolumn{21}{|l|}{ Ferns } \\
\hline Cbristella parasitica (L.) H. Lev. & & A & & & & & $x$ & & & $\mathrm{x}$ & & $\mathrm{x}$ & & & & & & & & \\
\hline Dicranopteris linearis (Burm. f.) Underwood & Uluhe; false staghorn fern & I & $\mathrm{x}$ & & $\mathrm{x}$ & $\mathrm{x}$ & $\mathrm{x}$ & $\mathrm{x}$ & $\mathrm{x}$ & $\mathrm{x}$ & & $\hat{\mathrm{x}}$ & $\mathrm{x}$ & $\mathrm{x}$ & $\mathrm{x}$ & $\mathrm{x}$ & $\mathrm{x}$ & $\mathrm{x}$ & $\mathrm{x}$ & $\mathrm{x}$ \\
\hline Nepbrolepis multiflora (Roxb.) Jarrett ex C. Morton & 'Okupukupu; sword fern & $\mathrm{A}$ & $\mathrm{x}$ & & $\mathrm{x}$ & $\mathrm{x}$ & & & $\mathrm{x}$ & $x$ & $\mathrm{x}$ & $\mathrm{x}$ & & & $\mathrm{x}$ & $\mathrm{x}$ & & & & \\
\hline Pblebodiumn aureum (L.) J. Sm. & Goldenfoot fern & $\mathrm{A}$ & & & & $\mathrm{x}$ & & & & & & & & & & & & & & \\
\hline Pbymatosorzus grossus (Langsd. \& Fisch.) Brownlie & Laua'e & $\mathrm{A}$ & & $\mathrm{x}$ & & & & & $\mathrm{x}$ & $\mathrm{x}$ & & & & & & & & & & \\
\hline Spbenomeris cbinensis (L.) Maxon & Pala'ā, lace fern & I & & $\mathrm{x}$ & & & & & & & & & $\mathrm{x}$ & & & $\mathrm{x}$ & $\mathrm{x}$ & & & \\
\hline \multicolumn{21}{|l|}{ Monocots } \\
\hline Freycinetia arborea Gaud. & 'Ie'ie & I & & & & $\mathrm{x}$ & & & & & & & & & & $\mathrm{x}$ & & & & \\
\hline Oplisnenus birtellus (L.) P. Beauv. & Honohono kukui; basket grass & $\mathrm{A}$ & & & & $\mathrm{x}$ & & & & & & & & & & & & & & \\
\hline Setaria palmifolia (J. Konig) Stapf & Palm grass & $\mathrm{A}$ & & & $\mathrm{x}$ & $\mathrm{x}$ & & & & & & $\mathrm{x}$ & & & & & & & & $\mathrm{x}$ \\
\hline Zingiber zerumbet (L.) Sm. & 'Awapuhi, shampoo ginger & $\mathbf{H}$ & & & & $\mathrm{x}$ & & & & & & & & & & & & & & \\
\hline \multicolumn{21}{|l|}{ Dicots } \\
\hline Acacia koa A. Gray & Koa & $\mathrm{E}$ & & & & & & $\mathrm{x}$ & $\mathrm{x}$ & $\mathrm{x}$ & $\mathrm{x}$ & $\mathrm{x}$ & $\mathrm{x}$ & $\mathrm{x}$ & $\mathrm{x}$ & & $\mathrm{x}$ & $\mathrm{x}$ & $\mathrm{x}$ & $\mathrm{x}$ \\
\hline Artbrostema ciliatum Pav. ex D. Don & & $\mathrm{A}$ & & & & & & & & $\mathrm{x}$ & & & & & & & & & & \\
\hline Casuarina equisetifolia $\mathrm{L}$. & Ironwood & $\mathrm{A}$ & $\mathrm{x}$ & & & & & & & & & & & & & & & & & \\
\hline Cecropia obtusifolia Bertol. & Guarumo & A & & & & & $\mathrm{x}$ & & & & & & & & & & & & & \\
\hline Centella asiatica (L.) Urb. & Pohe kula, Asiatic pennywort & $\mathrm{A}$ & & $\mathrm{x}$ & & & & & & & & & & & & & & & & \\
\hline Citbarexylum caudatum $\mathrm{L}$. & Fiddlewood & $\mathrm{A}$ & & & $\mathrm{x}$ & $\mathrm{x}$ & & $\mathrm{x}$ & $\mathrm{x}$ & $\mathrm{x}$ & & $\mathrm{x}$ & & & & $\mathrm{x}$ & $\mathrm{x}$ & & & \\
\hline Clidemia birta (L.) D. Don & Koster's curse & $\mathrm{A}$ & $\mathrm{x}$ & $\mathrm{x}$ & & & $\mathrm{x}$ & $\mathrm{x}$ & $x$ & $\mathrm{x}$ & $\mathrm{x}$ & $\mathrm{x}$ & & & & $x$ & $x$ & $\mathrm{x}$ & $\mathrm{x}$ & $\mathrm{x}$ \\
\hline Emilia soncbifolia $(\mathrm{L}.) \mathrm{DC}$ & Flora's paintbrush & A & & $\mathrm{x}$ & $\mathrm{x}$ & & & & & & & & & & & & & & & \\
\hline Erechtites valerianifolia (Wolf) DC & Fireweed & $\mathrm{A}$ & & & $\mathrm{x}$ & $\mathrm{x}$ & $\mathrm{x}$ & & & & & & & & $\mathrm{x}$ & & $\mathrm{x}$ & & $\mathrm{x}$ & \\
\hline Grevillea robusta A. Cunn. ex R. Br. & Silk oak; silver oak & $\mathrm{A}$ & $\mathrm{x}$ & & & & & & & & & & & & & & & & & \\
\hline Melaleuca quinquenervia (Cav.) S. T. Blake & Paperbark & $\mathrm{A}$ & & & & & & & & & & & & $\mathrm{x}$ & & & & & & \\
\hline Pandanus testorius S. Parkinson ex Z & Hala, pū hala & I & & & & $\mathrm{x}$ & & & & & & & & & & & & & & \\
\hline Passiflora edulis Sims. & Passion fruit & A & & & & $\mathrm{x}$ & & & & & & & & & & & & & & \\
\hline Psidium cattleianum Sabine & Strawberry guava & $\mathrm{A}$ & & & $\mathrm{x}$ & $\mathrm{x}$ & & & & & & & $\mathrm{x}$ & $\mathrm{x}$ & & & & & & \\
\hline Psidium guajava $\mathrm{L}$ & Guava & $\mathrm{A}$ & & & & & & $\mathrm{x}$ & & & & & & & & & & & & \\
\hline Rubus rosifolius Sm. & Thimbleberry & $\mathrm{A}$ & & & & & & $\mathrm{x}$ & & & & & & & & & & & & \\
\hline Rubus argutus Link & Blackberry & $\mathrm{A}$ & & & & & & & & & & & & & & & & $\mathrm{x}$ & $\mathrm{x}$ & \\
\hline Scbeffera actinopbylla (Endl.) Harms & Octopus tree & $\mathrm{A}$ & & $\mathrm{x}$ & & & & $\mathrm{x}$ & & & & & & & & & $\mathrm{x}$ & $\boldsymbol{\Lambda}$ & & \\
\hline Syzygium jambos (L.) Alston & Rose apple & $\mathrm{A}$ & $\mathrm{x}$ & & & & & & & & & & & & & & & & & \\
\hline
\end{tabular}

a, alien; E, endemic; I, indigenous; H, brought by Hawaiians colonizing the Islands.

1, Ironwood; 2, St. Stephen; 3, MC-steep; 4, MC-flat; 5, Púu Pia; 6, 'Aihualama; 7, 15 min; 8, MW-site A; 9, MW-site C; 10, 3 mile; 11, Guavakai 1; 12, Guavakai 2; 13, Powerline; 14, Mānoa Falls 1; 15, Mānoa Falls 2; 16, Mau'umae 1; 17, Mau'umae 2; 18, Mau'umae 3. 
TABLE 2

Percentage Cover from Quadrat and Transect Sampling of Predominant Plant Species Colonizing Permanent Plots after D. linearis Dieback

\begin{tabular}{|c|c|c|c|c|c|c|c|c|c|c|c|c|c|c|c|c|c|c|c|}
\hline \multirow[b]{3}{*}{ Site } & \multirow[b]{3}{*}{ Months } & \multicolumn{3}{|c|}{ Clidemia bista } & \multicolumn{3}{|c|}{ Nepbrolepis multiflora } & \multicolumn{3}{|c|}{ Setaria palmifolia } & \multicolumn{3}{|c|}{ Citbarexylum caudatum } & \multicolumn{3}{|c|}{ Dicranopteris linearis } & \multicolumn{3}{|c|}{ Acacia koa } \\
\hline & & \multicolumn{2}{|c|}{ Quadrat } & \multirow{2}{*}{$\begin{array}{c}\text { Transect } \\
\text { Mean } \\
\% \\
\text { Cover }\end{array}$} & \multicolumn{2}{|c|}{ Quadrat } & \multirow{2}{*}{ 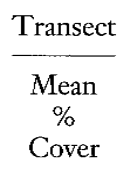 } & \multicolumn{2}{|c|}{ Quadrat } & \multirow{2}{*}{$\begin{array}{c}\text { Transect } \\
\text { Mean } \\
\% \\
\text { Cover }\end{array}$} & \multicolumn{2}{|c|}{ Quadrat } & \multirow{2}{*}{$\begin{array}{c}\text { Transect } \\
\text { Mean } \\
\% \\
\text { Cover }\end{array}$} & \multicolumn{2}{|c|}{ Quadrat } & \multirow{2}{*}{$\begin{array}{c}\text { Transect } \\
\text { Mean } \\
\% \\
\text { Cover }\end{array}$} & \multicolumn{2}{|c|}{ Quadrat } & \multirow{2}{*}{$\begin{array}{c}\text { Transect } \\
\text { Mean } \\
\% \\
\text { Cover }\end{array}$} \\
\hline & & $\begin{array}{c}\text { No. } \\
\text { of } \\
\text { Quads }\end{array}$ & $\begin{array}{c}\text { Mean } \\
\% \\
\text { Cover }\end{array}$ & & $\begin{array}{c}\text { No. } \\
\text { of } \\
\text { Quads }\end{array}$ & $\begin{array}{c}\text { Mean } \\
\% \\
\text { Cover }\end{array}$ & & $\begin{array}{c}\text { No. } \\
\text { of } \\
\text { Quads }\end{array}$ & $\begin{array}{c}\text { Mean } \\
\% \\
\text { Cover }\end{array}$ & & $\begin{array}{c}\text { No. } \\
\text { of } \\
\text { Quads }\end{array}$ & $\begin{array}{c}\text { Mean } \\
\% \\
\text { Cover }\end{array}$ & & $\begin{array}{c}\text { No. } \\
\text { of } \\
\text { Quads }\end{array}$ & $\begin{array}{c}\text { Mean } \\
\% \\
\text { Cover }\end{array}$ & & $\begin{array}{c}\text { No. } \\
\text { of } \\
\text { Quads }\end{array}$ & $\begin{array}{c}\text { Mean } \\
\% \\
\text { Cover }\end{array}$ & \\
\hline \multirow[t]{4}{*}{ Ironwood } & 0 & 7 & 3.6 & 11.9 & 1 & 0.2 & 1.2 & - & - & - & - & - & - & 7 & 12.9 & 7.1 & - & - & - \\
\hline & 6 & 7 & 16.8 & 14.3 & 2 & 0.6 & 2.4 & - & - & - & - & - & - & 7 & 20.5 & 7.1 & - & - & - \\
\hline & 14 & 8 & 17.5 & 27.4 & 1 & 0.1 & - & - & - & - & - & - & - & 7 & 16.0 & 13.1 & - & - & - \\
\hline & 31 & 6 & 15.8 & 40.5 & - & - & - & - & - & - & - & - & - & 8 & 22.5 & 7.1 & - & - & - \\
\hline \multirow[t]{4}{*}{ St. Stephen } & 0 & 2 & 0.2 & 2.4 & - & - & - & - & - & - & 3 & 0.4 & - & 10 & 30.9 & 45.2 & - & - & - \\
\hline & 6 & 6 & 1.6 & 1.2 & - & - & - & - & - & - & 4 & 1.0 & - & 10 & 48.8 & 45.2 & - & - & - \\
\hline & 14 & 7 & 1.0 & 1.2 & - & - & - & - & - & - & 5 & 1.0 & - & 10 & 44.5 & 59.5 & - & - & - \\
\hline & 27 & 7 & 2.4 & 1.2 & - & - & - & - & - & - & 4 & 1.6 & - & 10 & 81.0 & 70.2 & - & - & - \\
\hline \multirow[t]{4}{*}{ MC-steep } & 0 & - & - & 1.2 & - & - & - & - & - & - & 1 & 0.1 & - & 1 & 0.5 & - & 2 & 1.6 & 4.8 \\
\hline & 6 & - & - & - & - & - & - & - & - & - & 1 & 0.2 & - & 1 & 1.2 & 2.4 & 7 & 4.2 & 8.3 \\
\hline & 14 & - & - & - & - & - & 2.4 & - & - & 2.4 & 2 & 1.6 & 1.2 & 1 & 3.0 & 8.3 & 4 & 4.7 & - \\
\hline & 33 & 2 & 1.0 & - & 4 & 3.5 & 6.0 & 2 & 10.0 & 7.1 & 2 & 0.3 & 2.4 & 1 & 7.5 & 6.0 & 10 & 1.9 & 2.4 \\
\hline \multirow[t]{4}{*}{ MC-flat } & 0 & - & - & - & 6 & 14.2 & 15.5 & 1 & 1.0 & - & - & - & - & 9 & 29.5 & 60.7 & - & - & - \\
\hline & 6 & - & - & - & 6 & 14.5 & 13.1 & 3 & 8.5 & 1.2 & - & - & - & 9 & 43.1 & 64.3 & - & - & - \\
\hline & 14 & - & - & - & 6 & 12.6 & 11.9 & 2 & 6.2 & 15.5 & - & - & - & 9 & 49.0 & 58.3 & - & - & - \\
\hline & 32 & 2 & 1.0 & - & 6 & 4.8 & 3.6 & 4 & 5.7 & 7.1 & - & - & 3.6 & 10 & 60.0 & 64.3 & 1 & 0.1 & - \\
\hline \multirow[t]{4}{*}{ Púu Pia } & 0 & 7 & 13.9 & 13.1 & - & - & - & - & - & - & - & - & - & - & - & - & 3 & 1.1 & 1.2 \\
\hline & 6 & 8 & 14.7 & 34.5 & - & - & - & - & 一 & - & - & - & - & - & - & - & 5 & 6.5 & 3.6 \\
\hline & 14 & 9 & 16.3 & 35.7 & 1 & 0.1 & - & - & - & - & 2 & 0.5 & - & - & - & - & 4 & 6.3 & 1.2 \\
\hline & 32 & 8 & 13.6 & 40.5 & 2 & 0.2 & - & - & - & - & 7 & 1.1 & 9.5 & - & - & 1.2 & 6 & 7.1 & 3.6 \\
\hline \multirow[t]{4}{*}{ 'Aihualama } & 0 & 2 & 0.2 & - & - & - & - & - & - & - & - & - & - & 3 & 2.2 & 6.0 & 5 & 0.5 & 3.6 \\
\hline & 6 & 3 & 1.0 & - & - & - & - & - & 一 & - & 2 & 0.2 & - & 2 & 2.5 & 3.6 & 4 & 1.5 & 6.0 \\
\hline & 14 & 4 & 0.3 & 1.2 & - & - & - & - & - & - & 1 & 0.1 & 1.2 & 3 & 2.9 & 16.7 & 5 & 1.0 & 10.7 \\
\hline & 30 & 6 & 1.3 & 1.2 & - & - & - & - & - & - & 1 & 0.7 & - & 7 & 10.3 & 28.6 & 7 & 3.1 & 14.3 \\
\hline
\end{tabular}




\section{Wayside Vegetation Analysis}

TRANSECTS. The most common overstory species from quadrat sampling along the Maunawili Trail were the alien species $\mathrm{Aleu}$ rites moluccana, Psidium guajava, Psidium cattleianum, Cordyline fruticosa, and Citharexylum caudatum, with frequencies of $26,16,14,13$, and $8 \%$, respectively. The native Acacia koa was present in $5 \%$ of the quadrat samples. The most frequent plants in the understory were Clidemia birta, Oplismenus birtellus, D. linearis, Christella sp., Artbrostema sp., Rubus rosifolius, Nepbrolepis multiflora, Cordyline fruticosa, Paederia scandens, Setaria palmifolia, Citbarexylum caudatum, Sphenomeris chinensis, Zingiber zerumbet, Psidium cattleianum, and Cibotium splendens with frequencies of 54,45 , $26,24,23,22,20,19,18,14,9,8,8$, 7, and $6 \%$, respectively. Only $C$. splendens, D. linearis, and $S$. chinensis are native Hawaiian plants. Dead and live $D$. linearis were frequent and abundant along the Maunawili Trail; frequency of occurrence was 25 and $7.5 \%$ and mean percentage cover was 17 and $7 \%$ for live and dead $D$. linearis, respectively. The mean percentage cover for dead $D$. linearis when it was present in a quadrat was $95 \pm 10 \%$, whereas the mean percentage cover for live $D$. linearis was $66 \pm 37 \%$.

The most common overstory species along the 'Aiea Loop Trail were the alien species Eucalyptus sp., Eucalyptus robusta, and Psidium cattleianum, with quadrat frequencies of 35 , 26 , and $16 \%$, respectively. The native trees Metrosideros polymorpha, Cantbium odoratum, and Acacia koa were the next most common and had frequencies of 12,11 , and $10 \%$, respectively. The most frequent plants in the understory were Clidemia birta, Setaria palmifolia, D. linearis, Nepbrolepis multiflora, Blechnum occidentale, and Oplismenus birtellus, Spbenomeris chinensis, and Acacia koa seedlings, with frequencies of $59,33,25,16,11,8,5$, and $5 \%$, respectively. The four most common understory plants, $C$. birta, S. palmifolia, $D$. linearis, and $N$. multiflora, all tend to form large patches and dominate the understory when they occur. Although there was evidence of $D$. linearis dieback (dead stems beneath understory plants) along the 'Aiea Loop
Trail, nearly all the dead patches had fully revegetated.

A single transect at one elevation in each of two valleys is a limited sample with which to draw generalities about plant species composition. However, due to the steep slopes and thick vegetation these hiking trails were the only access available. Based on the two trail transects, $D$. linearis is estimated to occupy approximately $25 \%$ of the low-elevation valley slopes and ridgelines where we conducted our studies, and roughly $25 \%$ of the $D$. linearis patches were dead or showed signs of recent dieback.

DIEBACK PATCHES. The predominant plant species colonizing the 12 wayside sites were D. linearis, Clidemia birta, Acacia koa, and Nephrolepis multiflora, which occurred at 83, 75,75 , and $50 \%$ of the sites, respectively (Table 3). Average point-intercept frequencies for D. linearis, $C$. hirta, A. koa, and $N$. multiflora were $16.6,18.6,2.0$, and $25.5 \%$, respectively, at the sites where they were present. Considering both the 12 wayside and six permanent-plot dieback patches, $C$. birta and $N$. multiflora were the most frequent invaders, occurring at 63.2 and $42.1 \%$ of all sites, respectively, in March-June 1997.

\section{Mulch Plots}

The depth of dead $D$. linearis stems at the 'Aihualama, Mānoa Cliffs, and $\mathrm{Pu}^{\text {'u } u \text { Pia sites }}$ was $15.8,12.4$, and $8.4 \mathrm{~cm}$ for the thicket treatment; 6.6, 2.7, and $7.2 \mathrm{~cm}$ for the mulch treatment; and 0,0 , and $0 \mathrm{~cm}$ for the bare treatment, respectively. Because human disturbance made data collection impossible after 11 months for one of the replicates at the Mānoa Cliffs site, data from a 3-month census were used to make treatment comparisons. At all sites, seedlings of the primary revegetating species were most numerous in the bare treatment, intermediate in the mulch treatment, and lowest in the thicket treatment (Table 4). Analysis of variance on the mean number of Acacia koa seedlings showed a highly significant treatment effect at the 'Aihualama $(F=25.5, \mathrm{df}=2, P=0.001)$ and Mãnoa Cliffs $(F=16.6, \mathrm{df}=2, P=0.0001)$ sites. Analysis of variance on the mean num- 
TABLE 3

Predominant Plant Species Colonizing 12 Dead Dicranopteris linearis Patches on O'ahu and Kaua'i Determined by Transect Sampling, March-June 1997

\begin{tabular}{|c|c|c|c|c|c|c|c|c|c|c|}
\hline \multirow[b]{2}{*}{ Site } & \multirow[b]{2}{*}{ Island $^{a}$} & \multirow[b]{2}{*}{$n$} & \multirow[b]{2}{*}{$\mathrm{I}^{b}$} & \multicolumn{7}{|c|}{$\%$ Point Intercepts } \\
\hline & & & & $\begin{array}{c}\text { Clidemia } \\
\text { birta }\end{array}$ & Nepbrolepis & $\begin{array}{c}\text { Setaria } \\
\text { palmifolia }\end{array}$ & $\begin{array}{c}\text { Citbarexylem } \\
\text { caudatum }\end{array}$ & $\begin{array}{l}\text { Erechtites } \\
\text { valerianifolia }\end{array}$ & $\begin{array}{c}\text { Dicranopteris } \\
\text { linearis }\end{array}$ & $\begin{array}{c}\text { Acacia } \\
\text { koa }\end{array}$ \\
\hline $15 \mathrm{~min}$ & $\mathrm{Oa}$ & 63 & 62 & 1.6 & 39.7 & - & 1.6 & - & 42.9 & - \\
\hline$M W$-site A & $\mathrm{Oa}$ & 63 & 50 & 22.2 & 22.2 & - & 4.8 & - & 22.2 & 1.6 \\
\hline$M W$-site C & $\mathrm{Oa}$ & 63 & 63 & 36.5 & 58.7 & 1.6 & - & - & - & 3.2 \\
\hline 3 -mile & $\mathrm{Oa}$ & 63 & 43 & 23.8 & 1.6 & - & 1.6 & - & 38.1 & 1.6 \\
\hline Guavakai 1 & Ka & 84 & 15 & - & - & - & - & - & 8.3 & 3.6 \\
\hline Guavakai 2 & $\mathrm{Ka}$ & 84 & 26 & - & - & - & - & - & 25.0 & 1.2 \\
\hline Powerline & $\mathrm{Ka}$ & 84 & 44 & - & - & - & - & 1.2 & 3.6 & - \\
\hline Mānoa Falls 1 & $\mathrm{Oa}$ & 84 & 68 & 34.5 & 21.4 & - & 13.1 & 1.2 & 6.0 & $\rightarrow$ \\
\hline Mānoa Falls 2 & $\mathrm{Oa}$ & 84 & 23 & 16.7 & - & - & 3.6 & - & 2.4 & 1.2 \\
\hline Mau'umae 1 & $\mathrm{Oa}$ & 63 & 28 & 23.8 & 9.5 & - & - & - & - & 1.6 \\
\hline Mau'umae 2 & $\mathrm{Oa}$ & 63 & 28 & 7.1 & - & - & 一 & 3.6 & 13.1 & 1.2 \\
\hline Mau'umae 3 & $\mathrm{Oa}$ & 63 & 28 & 1.6 & - & 17.5 & - & - & 4.8 & 3.2 \\
\hline
\end{tabular}

"Oa, O'ahu; Ka, Kaua'i.

${ }^{b} \mathrm{I}$, number of intercepts from $n$ points.

TABLE 4

Germination of Seed Bank Plants in Three Simulated D. linearis Decomposition Treatments after 3 Months

\begin{tabular}{llrcc}
\hline & & \multicolumn{3}{c}{ No. of seedlings (mean $\pm \mathrm{SE})^{a}$} \\
\cline { 3 - 5 } Site & \multicolumn{1}{c}{ Species } & Bare & Mulch & Thicket \\
\hline 'Aihualama & Acacia koa & $38.7 \pm 8.4 \mathrm{a}$ & $8.3 \pm 4.8 \mathrm{~b}$ & $0.3 \pm 0.3 \mathrm{c}$ \\
& Clidemia birta & $3.7 \pm 2.7 \mathrm{a}$ & $0 \mathrm{a}$ & $0 \mathrm{a}$ \\
Mānoa Cliffs & Acacia koa & $15.7 \pm 4.1 \mathrm{a}$ & $9.3 \pm 4.8 \mathrm{~b}$ & $1.3 \pm 0.7 \mathrm{c}$ \\
& Erecbtites valerianifolia & $5.0 \pm 2.0 \mathrm{a}$ & $1.4 \pm 0.9 \mathrm{a}$ & $1.1 \pm 0.7 \mathrm{a}$ \\
Pu'u Pia & Casuarina equisetifolia & $9.0 \pm 6.1 \mathrm{a}$ & $4.0 \pm 3.5 \mathrm{~b}$ & $0.3 \pm 0.3 \mathrm{c}$ \\
& Dicranopteris linearis & $0.3 \pm 0.3 \mathrm{a}$ & $0 \mathrm{a}$ & $0 \mathrm{a}$ \\
\hline
\end{tabular}

${ }^{a}$ Means in a row followed by different letters are significantly different at $P<0.05$.

ber of ironwood seedings at the Pu'u Pia site was also highly significant $(F=16.6, \mathrm{df}=2$, $P=0.0001)$. Mean separations were significant for all treatment comparisons at all sites and invariably bare $>$ mulch $>$ thicket. The second most common revegetating species (Erechtites valerianifolia, Clidemia birta, or D. linearis) also was most abundant in the bare treatment at all sites; however, results of analysis of variance performed on the mean number of seedlings of the secondary species were not significant at any of the three sites.

\section{DISCUSSION}

Gap regeneration in native habitats has been studied for landslide, fire, and ungulate disturbance. Insect-induced disturbance is less well studied in natural systems in Hawai'i. The introduction of the leafhopper Sopbonia rufofascia has caused damage to the native fern D. linearis (Jones et al. 2000), and our data show that alien weeds colonize dead patches of fern. Several alien insect species are considered a threat to rare native plants (Follett 
et al. 2000, Howarth et al. 2001), but S. rufofascia was perhaps the first alien insect to cause widespread, highly visible damage to a common native plant (Fukada 1996, Jones et al. 2000). The invasion and infiltration of natural ecosystems by invasive insects is generally poorly documented except for intentional weed biological control releases.

Dieback patches typically occurred on sunny, open slopes (8-10 hr direct sun) (P.A.F., unpubl. data). Rarely did the area that had died extend into the shaded understory in monotypic stands of $D$. linearis or to open stands on the opposite side of the ridge where the angle of the sun was low $(2-3 \mathrm{hr}$ direct sun). Jones et al. (2000) showed in controlled studies that $S$. sopbonia damage to D. linearis progressed more rapidly in sunny sites compared with shaded sites. In addition to feeding damage, $S$. rufofascia typically oviposits into leaf midveins (Yang et al. 2000), disrupting vascular bundle integrity (Jones et al. 2000), which may reduce water transport and enhance symptoms of water stress in host plants. Yellow sticky-card traps used to monitor $S$. rufofascia poulations suggest that the leafhopper is more numerous or more active in sunny stands of $D$. linearis compared with shaded stands. For example, at the Ironwood site, paired traps placed in either direct sun or partial to full shade in adjacent stands of $D$. linearis were monitored biweekly for 2 months: sun traps caught an average of 66.2 adult leafhoppers whereas shade traps caught an average of $15.3(t=-5.24, \mathrm{df}=1,10$; $P=0.0004)$. Therefore, the pattern of dieback reflects microhabitat preferences of $S$. rufofascia (Jones et al. 2000), but may also be a function of differences in plant water relationships between exposed and shaded stands of D. linearis (e.g., Louda and Rodman 1996). Apparently the combination of factors leading to fern dieback has not been repeated because D. linearis is successfully reinvading dieback patches at five of six of the permanent plot sites (Table 3) in the presence of S. rufofascia.

Dead patches of $D$. linearis were colonized by both native and alien plant species; the number and assemblage of colonizing plant species was site specific. Colonists of dead patches usually reflected the plant community outside the boundaries of the patch. Most dieback patches were part of large monotypic stands of $D$. linearis, and therefore $D$. linearis was a common colonist. When mature Acacia koa trees were present near the dieback patch, koa seedlings were infrequently but consistently observed in areas where the thicket had decomposed sufficiently to allow light penetration to the soil surface. In fact, $D$. linearis dieback may fortuitously enhance regeneration of this once-dominant tree.

In both the permanent plots and wayside patch census Clidemia birta and Nepbrolepis multiflora were the most common invasive species colonizing and spreading in dieback patches. Clidemia birta is native to the West Indies and Central America, where it is an early colonizer of cleared ground and becomes dominant before it is smothered by gap-replacement vines (Smith 1992). It is a rare plant in its native range, however, only where it experiences heavy predation by insects and other organisms, and competition from other melastomes (Smith 1992). In Hawai'i, C. birta faces no competition from other melastomes and limited pressure from insect herbivores (Reimer and Beardsley 1989), and it is known to become a dominant plant after disturbances, creating monotypic stands (Cuddihy and Stone 1993). Most plants below the $C$. birta canopy disappear, even shade-tolerant species. When $C$. birta replaces the multilayered understory of native forests, their value as watersheds can be greatly diminished. Nepbrolepis multiflora is an aggressive, fast-growing fern native to India and tropical Asia. In Puerto Rico, it reportedly has become the most common fern on the island even though it was introduced only after 1940. It is an equally aggressive pioneer species in Hawai' $i$ and is commonly found in dense stands on roadsides, trail sides, and other disturbed sites (Wilson 1996).

The depth and density of dead $D$. linearis thickets and the rate of thicket decay will affect revegetation patterns. Woody plants and other seed-propagated species are generally unable to colonize thickets (MuellerDombois 2000) until the thickets have decayed to the mulch stage, which can take years at an undisturbed site. In parts of many 
of our plots, deep thickets were barren of plants except where footfalls had collapsed and broken $D$. linearis stems or otherwise exposed the soil. The undisturbed thicket treatment in the mulch plot experiment, where thicket depths were $8-12 \mathrm{~cm}$, produced greatly reduced seed germination compared with mulched stems and bare soil treatments. Dicranopteris linearis thickets can attain much greater depths in Hawai'i; at two of the permanent plots, 'Aihualama and St. Stephen, depths of dead fern thickets were estimated at $>50 \mathrm{~cm}$ throughout most of the plot, and thickets of 2-m depth are not uncommon in some areas (Kepler 1984). The habit of D. linearis of forming dense thickets favors reinvasion of dead patches by live $D$. linearis, colonizing vegetatively from the margins, rather than by seed bank species. Failure of seeded plants to survive is probably due to either the absence of favorable microsites for germination or a reduction in photosynthesis (Walker 1994). Also, D. linearis is known to show allelochemical inhibition of seedling growth (Aragon 1975). Nevertheless, dieback of $D$. linearis has generally created sites favorable to colonization by invasive weeds and thereby altered the gap dynamics in some of Hawai'i's rain forests.

\section{ACKNOWLEDGMENTS}

We thank V. Tanimoto (Hawai'i Department of Land and Natural Resources) for financial and operational support during this project. This work was supported by a McIntireStennis grant from the Hawai'i Department of Agriculture and the Hawai'i Department of Land and Natural Resources. Sandra Herrmann and Donna Ohora assisted in data collection. Mike McKenney assisted with Hawaiian plant names, and Cheryl Chan assisted with maps. Dieter Mueller-Dombois (Emeritus Professor, University of Hawai'i at Mānoa) provided a particularly helpful review of the paper.

\section{Literature Cited}

Aragon, E. L. 1975. Inhibitory effects of substances from residues and extracts of staghorn fern (Dicranopteris linearis). M.S. thesis, University of Hawai'i at Mānoa, Honolulu.

Atkinson, I. A. E. 1970. Successional trends in the coastal lowland forest of Mauna Loa and Kilauea Volcanoes, Hawaii. Pac. Sci. 24:387-400.

Ball, S. M. 2000. The hikers guide to O'ahu. Rev. ed. University of Hawai'i Press, Honolulu.

Cuddihy, L. W., and C. P. Stone. 1993. Alteration of native Hawaiian vegetation: Effects of humans, their activities and introductions. University of Hawai'i Press, Honolulu.

Follett, P. A., C. Empy-Campora, and V. P. Jones. 2000. Imidachloprid as a protectant for endangered plants attacked by Sophonia rufofascia. Proc. Hawaii. Entomol. Soc. 34:199-201.

Fukada, M. 1996. Distribution, host range, and seasonal history of the two-spotted leafhopper, Sophonia rufofascia (Kuoh and Kuoh), in Hawaii. M.S. thesis, University of Hawai'i at Mānoa, Honolulu.

Guariguata, M. R. 1990. Landslide disturbance and forest regeneration in the upper Luquillo Mountains of Puerto Rico. J. Ecol. 78:814-832.

Heu, R., and B. Kumashiro. 1989. Notes and exhibitions: Sophonia rufofascia. Proc. $\mathrm{Ha}$ waii. Entomol. Soc. 29:16-17.

Holttum, R. E. 1957. Morphology, growthhabit, and classification in the family Gleicheniaceae. Phytomorphology 7:168-184.

Howarth, F. G., G. M. Nishida, and N. L. Evenhuis. 2001. Insects and other arthropods. Pages 41-62 in G. W. Staples and R. H. Cowie, eds. Hawaii's invasive species. Bishop Museum, Honolulu, Hawai'i.

Jones, V. P., P. Anderson-Wong, P. A. Follett, P. Yang, D. Westcot, D. E. Ullman, J. Hu, and D. Foote. 2000. Feeding damage of the introduced leafhopper Sopbonia rufofascia (Homoptera: Cicadellidae) to plants in forests and watersheds of the Hawaiian Islands. Environ. Entomol. 29:171-180.

Kepler, A. K. 1984. Hawaiian heritage plants. Oriental Publication Company, Honolulu, Hawaici. 
Kochummen, K. M., and F. S. P. Ng. 1976. Natural plant succession after farming in Kepong. Malay. For. 40:61-78.

Louda, S. M., and J. F. Rodman. 1996. Insect herbivory as a major factor in the shade distribution of a native crucifer (Cardamine cordifolia A. Gray, bittercress). J. Ecol. 84:229-237.

Merlin, M. 1995. Hawaiian forest plants. Pacific Guide Books, Honolulu, Hawai'i.

Mueller-Dombois, D. 2000. Rain forest establishment and succession in the $\mathrm{Ha}$ waiian Islands. Landscape Urban Plann. 51:147-157.

Mueller-Dombois, D., and H. Ellenberg. 1974. Aims and methods in vegetation ecology. John Wiley \& Sons, New York.

Mueller-Dombois, D., and R. F. Fosberg. 1998. Vegetation of the tropical Pacific islands. Ecol. Stud. Vol. 132.

Palmer, D. D. 1993. Hawaiian ferns under attack. Fiddlehead 20:37.

Reimer, N. J., and J. W. Beardsley. 1989. Effectiveness of Liothrips urichi (Thysonoptera: Phlaeothripidae) introduced for biological control of Clidemia hirta in Hawaii. J. Econ. Entomol. 18:1141-1146.
SAS Institute. 2002. JMP user's guide. SAS Institute, Cary, North Carolina.

Scott, G. A. J. 1969. Relationship between vegetation and soil avalanching in the high rainfall areas of $\mathrm{O}^{\prime}$ ahu, Hawaii. M.S. thesis, University of Hawai' $i$ at Mānoa, Honolulu.

Smith, C. W. 1992. Distribution, status, phenology, rate of spread, and management of Clidemia in Hawaii. Pages 231-253 in C. P. Stone, C. W. Smith, and J. T. Tunison, eds. Alien plant invasions in native ecosystems of Hawaii. University of Hawai' $i$ Press, Honolulu.

Walker, L. R. 1994. Effects of fern thickets on woodland development on landslides in Puerto Rico. J. Veget. Sci. 5:525-532.

Wardlaw, C. W. 1931. Observations on the dominance of pteridophytes on some St. Lucia soils. J. Ecol. 19:60-63.

Wilson, K. A. 1996. Alien ferns in Hawai'i. Pac. Sci. 50:127-141.

Yang, P., P. A. Follett, V. P. Jones, and D. Foote. 2000. Oviposition behavior and egg parasitoids of Sophonia rufofascia (Homoptera: Cicadellidae) in Hawaii Volcanoes National Park. Proc. Hawaii. Entomol. Soc. 34:121-133. 